CERN-TH/96-261

hep-ph/9610362

\title{
Next to Leading Order QCD Corrections to Polarized $\Lambda$ Production in DIS
}

\author{
D. de Florian円 \\ Theoretical Physics Division, CERN, CH 1211 Geneva 23, Switzerland \\ e-mail: Daniel.de.Florian@cern.ch \\ R. Sassot \\ Departamento de Física, Universidad de Buenos Aires \\ Ciudad Universitaria, Pab.1 (1428) Bs.As., Argentina
}

\begin{abstract}
We calculate next to leading order QCD corrections to semi-inclusive polarized deep inelastic scattering and $e^{+} e^{-}$annihilation cross sections for processes where the polarization of the identified final-state hadron can also be determined. Using dimensional regularization and the HVBM prescription for the $\gamma_{5}$ matrix, we compute corrections for different spin-dependent observables, both in the $\overline{M S}$ and $\overline{M S_{p}}$ factorization schemes, and analyse their structure. In addition to the well known corrections to polarized parton distributions, we also present those for final-state polarized fracture functions and polarized fragmentation functions, in a consistent factorization scheme.
\end{abstract}

CERN-TH/96-261

October 1996

\footnotetext{
${ }^{1}$ On leave of absence from Departamento de Física, Universidad de Buenos Aires, Ciudad Universitaria Pab.1 (1428) Bs.As., Argentina.
} 


\section{Introduction}

In recent years, a considerable degree of attention has been paid to the semi-inclusive production of $\Lambda$ hyperons and their polarization. The approaches include experimental programmes to measure polarized fragmentation functions in $e^{+} e^{-} \rightarrow \Lambda+X$ [1] and semi-inclusive lepton-proton deep inelastic scattering (DIS) [2, 3], both in the current fragmentation region, as well as models for the production of these hyperons in existent $p \bar{p}$ and DIS experiments, but in the target fragmentation region [4]. Many of these studies are closely related to the interpretation of the proton spin structure [5, 6], due to the possibility of reconstructing it from the observed $\Lambda$ polarization.

Notwithstanding this increasing experimental interest in spin-dependent semi-inclusive $\Lambda$ physics, and also the important phenomenological insights related to the production of these hyperons in different processes, QCD corrections to such cross sections have received little attention. These corrections, however, have been shown to be crucial for the interpretation of totally inclusive polarized DIS experiments related to the spin structure of the proton [5, 6], and they certainly would have a non-negligible role in polarized $\Lambda$ production. On the other hand, one would naturally expect any parton model-inspired phenomenological description of the processes to mould into the more formal QCD-improved description, at least in some adequate limit. A description including fracture functions [7, 8] allows not only a consistent factorization of the collinear divergences characteristic of semi-inclusive processes, but also a perturbative picture of events where the identified finalstate hadron comes from the target fragmentation region.

In addition to their phenomenological interest, QCD corrections to polarized semi-inclusive processes involve some theoretical subtleties, such as factorization procedures [9], which in this case have to be extended to polarized fragmentation functions, and final-state polarized fracture functions, and which deserve a very careful treatment. In a recent paper [10] we have computed the $\mathcal{O}\left(\alpha_{s}\right)$ corrections to the one-particle inclusive polarized DIS cross sections, using dimensional regularization [11] and the HVBM [12] pre-

scription for the $\gamma_{5}$ matrix, discussing different factorization schemes. The analysis includes also target fragmentation effects by means of polarized fracture functions, but the polarization of the identified hadron is not taken into 
account, given that the observables under study are those related to pions and kaons in the final state.

In this paper we extend the approach of ref. [10] in order to include the possibility of polarized hadrons, such as the $\Lambda$, in the final state. This inclusion requires the computation of $\mathcal{O}\left(\alpha_{s}\right)$ corrections to polarized fragmentation functions, which dominate $\Lambda$ production in the current fragmentation region and can be measured in $e^{+} e^{-} \rightarrow \Lambda+X$, and also to final-state polarized fracture functions, which, as it has been said, account for target fragmentation effects and make possible a consistent factorization of collinear singularities in semi-inclusive DIS.

Next to leading order (NLO) corrections to polarized fragmentation functions have been addressed recently in refs. [13], using massive gluons to regulate infrared singularities. In this paper, however, we keep dimensional regularization and we propose a factorization prescription similar to the ones of our previous work [10, 14]. The factorization prescriptions for final-state polarized fracture functions can be generalized directly from those for polarized parton distributions, which guarantee the usual current conservation properties [15, 14]. The use of a common regularization procedure and natural factorization prescription allows a consistent and more direct analysis of cross sections for different processes.

In the following section we introduce the formal framework for obtaining the QCD corrections to polarized fragmentation functions in $e^{+} e^{-}$annihilation processes, and we specify our choice for factorization. In the third section we do the same for fracture functions, discussing there the structure of the different cross sections resulting from the combinations of polarization in the initial and final states. Finally, we summarize our results and present conclusions.

\section{2. $e^{+} e^{-}$annihilation}

In this section we obtain the NLO corrections for the semi-inclusive production of a longitudinally polarized hadron $(h)$ in $e^{+} e^{-}$annihilation. This process, besides offering the clearest way to define at leading order, and

eventually measure, the polarized fragmentation functions, allows us to show 
our choice for the factorization prescription, which defines the fragmentation functions beyond the leading order.

These corrections have recently been obtained in ref. [13], using off-shell gluons as regularization prescription. Since we want to make contact with previous calculations for other semi-inclusive DIS processes, which were performed using dimensional regularization, we first obtain the above-mentioned corrections using the latter prescription.

The usual kinematical variables used for the description of processes like the one under consideration, i.e. $e^{+}(l) e^{-}\left(l^{\prime}\right) \rightarrow h(p)+X$, are

$$
z=\frac{2 p \cdot q}{Q^{2}} \text { and } Q^{2}=q^{2}
$$

where the variable $q$ denotes the four-momentum of the virtual photon, and is defined by the momentum of the incoming leptons, $q=l+l^{\prime}$. In terms of these variables it is possible to write down the photon fragmentation tensor $W_{\mu \nu}$, whose antisymmetric part $W_{\mu \nu}^{A}$ is

$$
W_{\mu \nu}^{A}=2 \frac{i N_{c}}{z^{2} Q^{2}} \epsilon_{\mu \nu \rho \sigma} q^{\rho} s^{\sigma} g_{1}^{h}
$$

We do not include here the piece related to the additional semi-inclusive structure function $g_{2}^{h}$, which only contributes in the case of transverse polarization.

Taking the difference between the cross sections for the processes where the incoming electron and the outgoing hadron are polarized parallel and antiparallel to each other, we obtain

$$
\frac{d \sigma(\uparrow \uparrow-\downarrow \uparrow)}{d z d(\cos \theta)}=\alpha^{2} \frac{N_{c} \pi}{Q^{2}} g_{1}^{h} \cos \theta
$$

here $\theta$ is the angle between the hadron and the beam directions.

In the naive parton model, the polarized structure function $g_{1}^{h}(z)$ is given by the convolution between the partonic cross section corresponding to the Feynman diagram of fig. 1a and the polarized semi-inclusive fragmentation functions

$$
\Delta D_{h / q}(z)=D_{q \uparrow}^{h \uparrow}(z)-D_{q \downarrow}^{h \uparrow}(z)
$$


i.e.

$$
g_{1}^{h}(z)=\sum_{q}^{N_{f}} e_{q}^{2}\left[\Delta D_{h / q}(z)+\Delta D_{h / \bar{q}}(z)\right] .
$$

In NLO of QCD, the interference between the diagrams of figs. 1a and $1 \mathrm{~b}$, that between the first one and those in $1 \mathrm{c}$ and $1 \mathrm{~d}$, as well as the whole contributions from the diagrams of fig. 2 are included, in both cases taking into account that either quarks or gluons undergo hadronization. Then, eq. (5) develops the less trivial convoluted expression:

$$
\begin{array}{r}
g_{1}^{h}\left(z, Q^{2}\right)=\sum_{q}^{N_{f}} e_{q}^{2} \int_{z}^{1} \frac{d y}{y}\left\{\left[\delta(1-y)+\Delta C_{q}\left(y, \frac{Q^{2}}{\mu^{2}}\right)\right] \times\right. \\
\left.\left[\Delta D_{h / q}\left(\frac{z}{y}\right)+\Delta D_{h / \bar{q}}\left(\frac{z}{y}\right)\right]+\Delta C_{g}\left(y, \frac{Q^{2}}{\mu^{2}}\right) \Delta D_{h / g}\left(\frac{z}{y}\right)\right\} .
\end{array}
$$

Since in some intermediate steps of the calculation, both infrared and soft collinear divergences appear, (the UV divergences can be avoided by working in the Landau gauge), it is necessary to define a prescription to isolate them. For this purpose, and throughout this paper, we use dimensional regularization and the HVBM scheme, which allows us to deal with typically four-dimensional objects such as $\epsilon^{\mu \nu \rho \sigma}$ and $\gamma^{5}$ in a fully consistent way. As is well known [9, 16], spurious terms appear in this scheme because of the breaking of the chiral symmetry. These terms can be absorbed in the factorization procedure in order to keep the naive interpretation of the first moment of the polarized fragmentation function, i.e. as the fraction of the spin of the parton carried by the outgoing hadron.

In order to calculate the NLO corrections, it is useful to define the variables $x_{i}=2 k_{i} \cdot q / Q^{2}=2 E_{i} / Q$, in terms of the momentum carried by the three-final state partons. For the sake of simplicity, we choose the $z$ direction as that of the parton labelled by the index 1 , and $\phi$ as the angle between the parton labelled 2 and the $z$ direction. Momentum conservation implies that

$$
x_{2}=\frac{1-x_{1}}{1-x_{1}(1-y)},
$$

where

$$
y=\frac{1+\cos \phi}{2},
$$


and integrating over the particles labelled 2 and 3 (particle 1, which undergoes hadronisation, is not integrated), the three $(2+1)$ particle phase space reduces to

$$
P S^{(2+1)}=\frac{(4 \pi)^{\epsilon}}{8 \pi} \int_{0}^{1} d y \frac{x_{1}^{1-2 \epsilon}}{1-x_{1}} x_{2}^{2} \int_{0}^{Q^{2} x_{2}^{2} y(1-y)} d|\hat{k}|^{2} \frac{|\hat{k}|^{-2(1+\epsilon)}}{\Gamma(-\epsilon)} .
$$

Here $|\hat{k}|^{2}$ is the square of the $d-4=-2 \epsilon$ dimensional component of the momentum of the outgoing (integrated) particles 2 and 3.

Projecting conveniently the matrix elements corresponding to the diagrams in fig. 2, and integrating over the phase space, we obtain the real emission contributions to $\Delta C_{q}$ (we label the outgoing quark as number 1):

$$
\begin{aligned}
\Delta C_{q}\left(y, \frac{Q^{2}}{\mu^{2}}\right) & =\left(\frac{4 \pi \mu^{2}}{Q^{2}}\right)^{\epsilon} \frac{\Gamma(1-\epsilon)}{\Gamma(1-2 \epsilon)} \frac{\alpha_{s}}{2 \pi} C_{F}\left\{-\frac{1}{\epsilon}\left[\frac{3}{2} \delta(1-y)+\frac{1+y^{2}}{(1-y)_{+}}\right]\right. \\
& \left.+\delta(1-y)\left[\frac{2}{\epsilon^{2}}+\frac{3}{\epsilon}+8-\frac{2}{3} \pi^{2}\right]+\Delta f_{q}^{D}(y)\right\}
\end{aligned}
$$

with

$$
\begin{aligned}
\Delta f_{q}^{D}(y) & =\delta(1-y)\left[\frac{2}{3} \pi^{2}-\frac{9}{2}\right]-\frac{3}{2}\left(\frac{1}{1-y}\right)_{+}+\left(1+y^{2}\right)\left(\frac{\ln (1-y)}{1-y}\right)_{+} \\
& +2 \frac{1+y^{2}}{1-y} \ln y-\frac{3}{2}(1-y)-2(\widehat{1-y}) .
\end{aligned}
$$

In the last equation, we have kept track of terms originated in the $4-2 \epsilon$ dimensional momentum integration, writing them under hats just for factorization purposes. The second term in the r.h.s. of eq. (10), which contains the infrared divergences, cancels identically when adding the virtual contributions coming from the interference of diagrams in fig. 1, as is generally expected.

The same procedure can be applied in order to obtain the gluonic coefficient (now, labelling the gluon as parton 1) obtaining

$$
\Delta C_{g}\left(y, \frac{Q^{2}}{\mu^{2}}\right)=\left(\frac{4 \pi \mu^{2}}{Q^{2}}\right)^{\epsilon} \frac{\Gamma(1-\epsilon)}{\Gamma(1-2 \epsilon)} \frac{\alpha_{s}}{2 \pi} C_{F}\left\{-\frac{1}{\epsilon}[2(2-y)]+\Delta f_{g}^{D}(y)\right\},
$$


with

$$
\Delta f_{g}^{D}(y)=2\left\{(2-y) \ln \left[(1-y) y^{2}\right]-(2-y)-2(\widehat{1-y})\right\}
$$

Having computed the whole cross section up to next to leading order, we are now able to factorize the divergences by means of the definition of scaledependent polarized fragmentation functions $\left(\Delta D_{h / q}\left(z, Q^{2}\right)\right.$ and $\left.\Delta D_{h / g}\left(z, Q^{2}\right)\right)$. Fixing the factorization scale $\mu^{2}$ equal to $Q^{2}$, in the $\overline{M S}$ scheme the prescription amounts to absorbing only the $1 / \hat{\epsilon}$ terms. It has been shown that within the HVBM method this prescription leaves unsubtracted some soft finite contributions directly related to the hat terms. It is also frequent to define a different scheme, called $\overline{M S_{p}}$ [15], where these contributions are subtracted. The general expression for the distributions, whithout specifying any scheme, is then given by

$$
\begin{gathered}
\Delta D_{h / q}(z)=\int_{z}^{1} \frac{d y}{y}\left\{\left[\delta(1-y)+\frac{\alpha_{s}}{2 \pi}\left(\frac{1}{\hat{\epsilon}} \Delta P_{q \leftarrow q}(y)-C_{F} \Delta \tilde{f}_{q}^{D}(y)\right)\right] \times\right. \\
\left.\Delta D_{h / q}\left(\frac{z}{y}, Q^{2}\right)+\left(\frac{1}{\hat{\epsilon}} \Delta P_{g \leftarrow q}(y)-C_{F} \Delta \tilde{f}_{g}^{D}(y)\right) \Delta D_{h / g}\left(\frac{z}{y}, Q^{2}\right)\right\},
\end{gathered}
$$

where

$$
\frac{1}{\hat{\epsilon}} \equiv \frac{1}{\epsilon} \frac{\Gamma[1-\epsilon]}{\Gamma[1-2 \epsilon]}\left(\frac{4 \pi \mu^{2}}{Q^{2}}\right)^{\epsilon}=\frac{1}{\epsilon}-\gamma_{E}+\log 4 \pi+\log \frac{\mu^{2}}{Q^{2}}+\mathcal{O}(\epsilon)
$$

In the $\overline{M S_{p}}$ scheme, the finite subtraction terms $\Delta \tilde{f}_{q}^{D}(y)=\Delta \tilde{f}_{g}^{D}(y)=$ $4(y-1)$ are designed to absorb the soft contributions coming from the real gluon emission diagrams in fig. 2 and, therefore, they are the same as the one obtained for the definition of NLO polarized quark distributions in DIS. In fact, the hat terms obtained for the real gluon emission diagrams in DIS are equal to the ones obtained in this analysis.

Using the former definition for NLO distributions, the final expression for the semi-inclusive structure function reads

$$
\begin{array}{r}
g_{1}^{h}\left(z, Q^{2}\right)=\sum_{q}^{N_{f}} e_{q}^{2} \int_{z}^{1} \frac{d y}{y}\left\{\left[\delta(1-y)+\frac{\alpha_{s}}{2 \pi} C_{F}\left(\Delta f_{q}^{D}(y)-\Delta \tilde{f}_{q}^{D}(y)\right)\right] \times\right. \\
\left.\left[\Delta D_{h / q}\left(\frac{z}{y}\right)+\Delta D_{h / \bar{q}}\left(\frac{z}{y}\right)\right]+\frac{\alpha_{s}}{2 \pi} C_{F}\left[\Delta f_{g}^{D}(y)-2 \Delta \tilde{f}_{g}^{D}(y)\right] \Delta D_{h / g}\left(\frac{z}{y}\right)\right\}
\end{array}
$$


where, as usual, in the $\overline{M S}$ scheme the finite subtracted terms are chosen to be zero.

Notice that although the full expression for the cross sections, and the definition of scale-dependent densities, differs from those of refs. [13] in the finite terms, the evolution kernels, which are not scheme-dependent, are identical, as expected.

\section{Semi-Inclusive Deep Inelastic Scattering}

With the definition for polarized fragmentation functions given in the previous section, eq. (14), we are now able to compute the NLO corrections for semi-inclusive DIS in the case in which the final-state hadron is polarized. NLO contributions for processes with unpolarized final-state hadrons (with either polarized or unpolarized initial states) have been computed in refs. [10] and [8], respectively, so we refer the reader to these for most of the definitions and conventions.

Using the usual kinematical DIS variables for the interaction between a lepton of momentum $l$ and helicity $\lambda_{l}$ and a nucleon $A$ of momentum $P$ and helicity $\lambda_{A}$

$$
x=\frac{Q^{2}}{2 P \cdot q}, \quad y=\frac{P \cdot q}{P \cdot l}, \quad Q^{2}=-q^{2} \text { and } S_{H}=(P+l)^{2},
$$

the differential cross section for the production of a hadron $h$ with energy $E_{h}=z E_{A}(1-x)$ and helicity $\lambda_{h}$ (with $n$ partons in the final state) can be written as

$$
\begin{aligned}
\frac{d \sigma^{\lambda_{l} \lambda_{A} \lambda_{h}}}{d x d y d z} & =\int \frac{d u}{u} \sum_{n} \sum_{j=q, \bar{q}, g} \int d P S^{(n)} \frac{\alpha^{2}}{S_{H} x} \frac{1}{e^{2}(2 \pi)^{2 d}} \\
& \times\left[Y_{M}\left(-g^{\mu \nu}\right)+Y_{L} \frac{4 x^{2}}{Q^{2}} P_{\mu} P_{\nu}+\lambda_{l} Y_{P} \frac{x}{Q^{2}} i \epsilon^{\mu \nu q P}\right] \\
& \times \sum_{\lambda_{1}, \lambda_{2}= \pm 1} H_{\mu \nu}\left(\lambda_{1}, \lambda_{2}\right)\left\{M_{j, h / A}\left(\frac{x}{u}, \frac{E_{h}}{E_{A}}, \frac{\lambda_{1}}{\lambda_{A}}, \frac{\lambda_{h}}{\lambda_{A}}\right)(1-x)\right. \\
& \left.+f_{j / A}\left(\frac{x}{u}, \frac{\lambda_{1}}{\lambda_{A}}\right) \sum_{i_{\alpha}=q, \bar{q}, g} D_{h / i_{\alpha}}\left(\frac{z}{\rho}, \frac{\lambda_{h}}{\lambda_{2}}\right) \frac{1}{\rho}\right\}
\end{aligned}
$$


where $\rho=E_{\alpha} / E_{A}$. The helicity-dependent partonic tensor is defined by

$$
H_{\mu \nu}\left(\lambda_{1}, \lambda_{2}\right)=M_{\mu}\left(\lambda_{1}, \lambda_{2}\right) M_{\nu}^{\dagger}\left(\lambda_{1}, \lambda_{2}\right),
$$

where $M_{\mu}$ is the parton-photon matrix element with the photon polarization vector subtracted. Here $\lambda_{1}$ and $\lambda_{2}$ are the helicities of the initial- and final-state partons respectively. Both $f_{j / A}$ and $D_{h / i_{\alpha}}$ are the usual parton distribution and fragmentation functions, respectively, which represent the probabilities of finding a parton $j$ or a hadron $h$ with a given momentum fraction and helicity with respect from the parent particle (the target $A$ or the parton $i_{\alpha}$, respectively).

As was shown in ref. [7], it is necessary to introduce a new distribution for semi-inclusive processes, the fracture function, which gives the probability of finding a polarized parton $j$ and a polarized hadron $h$ in the nucleon, denoted by $M_{j, h / A}\left(\frac{x}{u}, \frac{E_{h}}{E_{A}}, \frac{\lambda_{1}}{\lambda_{A}}, \frac{\lambda_{h}}{\lambda_{A}}\right)$. Including them, it will be possible to factorize all the collinear divergences that remain in the NLO contributions after the redefinition of the scale-dependent parton distribution and fragmentation functions.

Taking different linear combinations of cross sections for targets and finalstate hadrons with equal or opposite helicities, it is possible to isolate parton distributions, fragmentation functions and fracture functions of different kind and their QCD corrections. For example, taking the sum over all the helicity states

$$
\sigma=\sigma^{\lambda_{l}++}+\sigma^{\lambda_{l}+-}+\sigma^{\lambda_{l}-+}+\sigma^{\lambda_{l}--},
$$

the result is proportional to a convolution of unpolarized parton distributions,

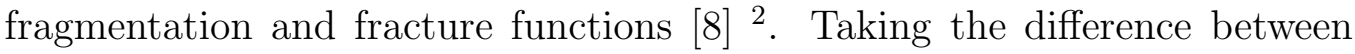
cross sections with opposite target helicities, as in ref. [10]:

$$
\Delta \sigma=\left(\sigma^{\lambda_{l}++}+\sigma^{\lambda_{l}+-}\right)-\left(\sigma^{\lambda_{l}-+}+\sigma^{\lambda_{l}--}\right),
$$

the result contains polarized parton distributions, single polarized fracture functions (single initial-state polarization), and unpolarized fragmentation functions. In the following two subsections, we consider the two remaining

\footnotetext{
${ }^{2}$ Notice the slight difference in the normalization used here (a factor of 2) with respect to ref. [8], where an average over the polarizations of the target was taken.
} 
possibilities, which represent the case where only the final-state hadron polarization is put in evidence (single final-state polarization) at cross section level:

$$
\sigma \Delta=\left(\sigma^{\lambda_{l}++}-\sigma^{\lambda_{l}+-}\right)+\left(\sigma^{\lambda_{l}-+}-\sigma^{\lambda_{l}--}\right),
$$

and that where both polarizations are relevant (double polarization):

$$
\Delta \sigma \Delta=\left(\sigma^{\lambda_{l}++}-\sigma^{\lambda_{l}+-}\right)-\left(\sigma^{\lambda_{l}-+}-\sigma^{\lambda_{l}--}\right) .
$$

\section{3a. Single (final-state) polarization}

It is straightforward to show that the single (final-state) polarization cross section $\sigma \Delta$ is proportional to the convolutions

$$
\begin{aligned}
& \sigma \Delta \propto f_{j / A}(x / u) \otimes H \Delta(u, \rho) \otimes \Delta D_{h / i_{\alpha}}(z / \rho) \\
& \quad+(1-x) M \Delta_{j, h / A}(x / u,(1-x) z) \otimes \Delta H^{\prime}(u)
\end{aligned}
$$

where the first term corresponds to current fragmentation processes and the second to fragmentation of the target. In the former, $f_{j / A}(x / u)$ are just the unpolarized parton distributions, $\Delta D_{h / i_{\alpha}}(z / \rho)$ are the polarized fragmentation functions defined in section 2 , and $H \Delta=\sum_{\lambda_{1}, \lambda_{2}} \lambda_{2} H\left(\lambda_{1}, \lambda_{2}\right)$ is the partonic tensor for polarized final states. The convolutions are those defined

by eq. (18). In the second term of eq. (24) we have defined the final state polarized fracture function as

$$
M \Delta=M_{j, h / A}(+,+)+M_{j, h / A}(-,-)-M_{j, h / A}(+,-)-M_{j, h / A}(-,+),
$$

where we have omitted the first two kinematical arguments in the fracture functions, and the single polarized tensor $\Delta H^{\prime}=\sum_{\lambda_{1}, \lambda_{2}} \lambda_{1} H\left(\lambda_{1}, \lambda_{2}\right)$ (integrated in $\rho$ ).

Computing the matrix elements of the diagrams in figs. 4, 5 and 6 , contracting with the adequate projector

$$
P_{p o l}^{\mu \nu} \equiv \frac{\alpha^{2}}{S_{H} x} \frac{1}{e^{2}(2 \pi)^{2 d}} \frac{x}{Q^{2}} i \epsilon^{\mu \nu q P},
$$


and integrating them over the phase space as it done in ref. [10], we finally find:

$$
\begin{aligned}
& \frac{d \sigma \Delta}{d x d y d z}=Y^{p} \lambda_{l} \sum_{i=q, \bar{q}} c_{i}\left\{\int \int _ { A } \frac { d u } { u } \frac { d \rho } { \rho } \left\{q_{i}\left(\frac{x}{u}\right) \Delta D_{q_{i}}\left(\frac{z}{\rho}\right) \delta(1-u) \delta(1-\rho)\right.\right. \\
+ & q_{i}\left(\frac{x}{u}\right) \Delta D_{q_{i}}\left(\frac{z}{\rho}\right) \\
& \times \frac{\alpha_{s}}{2 \pi}\left[-\frac{1}{\hat{\epsilon}}\left(\Delta P_{q \leftarrow q}(\rho) \delta(1-u)+P_{q \leftarrow q}(u) \delta(1-\rho)\right)+C_{f} \Phi \Delta_{q q}(u, \rho)\right] \\
+ & q_{i}\left(\frac{x}{u}\right) \Delta D_{g}\left(\frac{z}{\rho}\right) \\
& \times \frac{\alpha_{s}}{2 \pi}\left[-\frac{1}{\hat{\epsilon}}\left(\Delta P_{g \leftarrow q}(\rho) \delta(1-u)+\hat{P} \Delta_{g q \leftarrow q}(u) \delta(\rho-a)\right)+C_{f} \Phi \Delta_{q g}(u, \rho)\right] \\
+ & g\left(\frac{x}{u}\right) \Delta D_{q_{i}}\left(\frac{z}{\rho}\right) \\
\times & \left.\frac{\alpha_{s}}{2 \pi}\left[-\frac{1}{\hat{\epsilon}}\left(P_{q \leftarrow g}(u) \delta(1-\rho)+\hat{P} \Delta_{q \bar{q} \leftarrow g}(u) \delta(\rho-a)\right)+T_{f} \Phi \Delta_{g q}(u, \rho)\right]\right\} \\
+ & M \Delta_{q_{i}}\left(\frac{x}{u},(1-x) z\right) \frac{\alpha_{s}}{2 \pi}\left[-\frac{1}{\hat{\epsilon}} \Delta P_{q \leftarrow q}(u)+C_{f} \Phi \Delta_{q}(u)\right] \\
+ & \left.\left.M \Delta_{g}\left(\frac{x}{u},(1-x) z\right) \frac{\alpha_{s}}{2 \pi}\left[-\frac{1}{\hat{\epsilon}} \Delta P_{q \leftarrow g}(u)+T_{f} \Phi \Delta_{g}(u)\right]\right\}\right\}
\end{aligned}
$$

where the coefficients $\Phi \Delta_{i j}$ are given in the Appendix,

$$
c_{j}=4 \pi Q_{q_{j}}^{2} \frac{\alpha^{2}}{S_{H} x},
$$

and the integration limits can be found in ref. [10].

The poles proportional to $\delta(1-u)$ and to the polarized Altarelli-Parisi kernels $\Delta P_{i \leftarrow j}$ [17] correspond to final-state singularities and are subtracted with the definition of the NLO polarized fragmentation functions given in section 2. The coefficients $\Phi \Delta_{i j}$ contain finite soft terms (proportional to the terms marked with hats) related to polarized final-states which are also subtracted by means of eq. (14) in the $\overline{M S_{p}}$ scheme. 
Those poles proportional to $\delta(1-\rho)$ and to the unpolarized kernels are related to the initial-state singularities and are therefore subtracted by the NLO definition of unpolarized parton distributions. In this case both the $\overline{M S}$ and the $\overline{M S_{p}}$ schemes coincide, since we are dealing here with unpolarized initial-state processes, so there are no finite soft terms. Notice that there are no hat terms proportional to $\delta(1-\rho)$ in the coefficients.

Finally, the poles proportional to $\delta(\rho-a)$, where $a=x(1-u) / u(1-x)$, represent configurations where the hadrons are produced by the current but in the direction of the incoming nucleon. These are subtracted along with the target-initiated singularities (homogeneous) by the NLO definition of final-state polarized fracture functions

$$
\begin{aligned}
& M \Delta_{q_{i}}(\xi, \zeta)= \\
& \int_{\frac{\xi}{1-\zeta}}^{1} \frac{d u}{u}\left\{\left[\delta(1-u)+\frac{\alpha_{s}}{2 \pi}\left(\frac{1}{\hat{\epsilon}} \Delta P_{q \leftarrow q}(u)-C_{f} \Delta \tilde{f}_{q}^{M H}(u)\right)\right] M \Delta_{q_{i}}\left(\frac{\xi}{u}, \zeta, Q^{2}\right)\right. \\
&\left.+\frac{\alpha_{s}}{2 \pi}\left[\frac{1}{\hat{\epsilon}} \Delta P_{q \leftarrow g}(u)-T_{f} \Delta \tilde{f}_{g}^{M H}(u)\right] M \Delta_{g}\left(\frac{\xi}{u}, \zeta, Q^{2}\right)\right\} \\
&+\int_{\xi}^{\frac{\xi}{\xi+\zeta}} \frac{d u}{u} \frac{u}{x(1-u)}\left\{\frac{\alpha_{s}}{2 \pi}\left[\frac{1}{\hat{\epsilon}} \hat{P} \Delta_{g q \leftarrow q}(u)-C_{f} \Delta \tilde{f}_{q}^{M I}(u)\right]\right. \\
& \times q_{i}\left(\frac{\xi}{u}, Q^{2}\right) \Delta D_{g}\left(\frac{\zeta u}{\xi(1-u)}, Q^{2}\right) \\
&+\frac{\alpha_{s}}{2 \pi}\left[\frac{1}{\hat{\epsilon}} \hat{P} \Delta_{q \bar{q} \leftarrow g}(u)-\frac{\alpha_{s}}{2 \pi} T_{f} \Delta \tilde{f}_{g}^{M I}(u)\right] \\
&\left.\times g\left(\frac{\xi}{u}, Q^{2}\right) \Delta D_{q_{i}}\left(\frac{\zeta u}{\xi(1-u)}, Q^{2}\right)\right\}
\end{aligned}
$$

where the homogeneous $(M H)$ and inhomogeneous $(M I)$ counterterms in the $\overline{M S_{p}}$ are given by

$$
\begin{aligned}
\Delta \tilde{f}_{q}^{M I}(u) & =\Delta \tilde{f}_{q}^{M H}(u)=4(u-1) \\
\Delta \tilde{f}_{g}^{M I}(u) & =\Delta \tilde{f}_{g}^{M H}(u)=2(1-u) .
\end{aligned}
$$

These are identical to those in the final-state polarized case, since the structure of the corrections is the same (actually, the homogeneous coefficients are exactly the same). 
One interesting feature of the divergences is that the poles corresponding to $\delta(1-\rho)$ and $\delta(\rho-a)$ in eq. (27) differ only in a global $\operatorname{sign}\left(-\hat{P} \Delta_{q \bar{q} \leftarrow g}(x)=\right.$ $\left.P_{q \leftarrow g}(x)\right)$. This sign accounts for the opposite polarizations of the quark in the $\gamma g \rightarrow q \bar{q}$ process according to whether the quark is emitted in the photon direction $(\rho=1)$ or in the opposite configuration $(\rho=a)$.

Applying the factorization procedure, we obtain the following cross section

$$
\begin{aligned}
& \frac{d \sigma \Delta}{d x d y d z}= \\
& Y^{p} \lambda_{l} \sum_{i=q, \bar{q}} c_{i}\left\{\int \int _ { A } \frac { d u } { u } \frac { d \rho } { \rho } \left\{q_{i}\left(\frac{x}{u}, Q^{2}\right) \Delta D_{q_{i}}\left(\frac{z}{\rho}, Q^{2}\right) \delta(1-u) \delta(1-\rho)\right.\right. \\
&+ q_{i}\left(\frac{x}{u}, Q^{2}\right) \Delta D_{q_{i}}\left(\frac{z}{\rho}, Q^{2}\right) \frac{\alpha_{s}}{2 \pi} C_{f}\left[\Phi \Delta_{q q}(u, \rho)-\Delta \tilde{f}_{q}^{D}(\rho) \delta(1-u)\right] \\
&+ q_{i}\left(\frac{x}{u}, Q^{2}\right) \Delta D_{g}\left(\frac{z}{\rho}, Q^{2}\right) \\
& \times \frac{\alpha_{s}}{2 \pi} C_{f}\left[\Phi \Delta_{q g}(u, \rho)-\Delta \tilde{f}_{g}^{D}(\rho) \delta(1-u)-\Delta \tilde{f}_{q}^{M I}(u) \delta(\rho-a)\right] \\
&+\left.g\left(\frac{x}{u}, Q^{2}\right) \Delta D_{q_{i}}\left(\frac{z}{\rho}, Q^{2}\right) \frac{\alpha_{s}}{2 \pi} T_{f}\left[\Phi \Delta_{g q}(u, \rho)-\Delta \tilde{f}_{g}^{M I}(u) \delta(\rho-a)\right]\right\} \\
&+\int_{B} \frac{d u}{u}(1-x)\left\{\Delta _ { q _ { i } } \left(\frac{x}{u},(1-x) z, Q_{q_{i}}\left(\frac{x}{u},(1-x) z, Q^{2}\right) \delta(1-u)\right.\right. \\
&+ M \Delta_{g}\left(\frac{x}{u},(1-x) z, Q_{f}^{2}\right) \frac{\alpha_{s}}{2 \pi} T_{f}\left[\Phi \Delta_{q}(u)-\Delta \tilde{f}_{q}^{M H}(u)\right]
\end{aligned}
$$

which is free of collinear divergences.

\section{3b. Double (initial- and final-state) Polarization}

An analysis similar to that of subsection 3.a shields for $\Delta \sigma \Delta$ the following convolutions

$$
\begin{aligned}
\Delta \sigma \Delta & \propto \Delta f_{j / A}(x / u) \otimes \Delta H \Delta(u, \rho) \otimes \Delta D_{h / i_{\alpha}}(z / \rho) \\
& +(1-x) \Delta M \Delta_{j, h / A}(x / u,(1-x) z) \otimes H^{\prime}(u),
\end{aligned}
$$


where again the first term corresponds to current fragmentation processes and the second to fragmentation of the target. In the former, $\Delta f_{j / A}(x / u)$ are now polarized parton distributions, $\Delta D_{h / i_{\alpha}}(z / \rho)$ are polarized fragmentation functions, and $\Delta H \Delta=\sum_{\lambda_{1}, \lambda_{2}} \lambda_{1} \lambda_{2} H\left(\lambda_{1}, \lambda_{2}\right)$ is the partonic tensor for polarized initial and final states. In the second term of eq. (32) we define the doubly polarized fracture functions as

$$
\Delta M \Delta=M_{j, h / A}(+,+)-M_{j, h / A}(-,-)-M_{j, h / A}(+,-)+M_{j, h / A}(-,+),
$$

and tensor $H^{\prime}=\sum_{\lambda_{1}, \lambda_{2}} H\left(\lambda_{1}, \lambda_{2}\right)$, also integrated in $\rho$.

Using now the metric and longitudinal projectors

$$
P_{M}^{\mu \nu}=-g^{\mu \nu} \quad \text { and } \quad P_{L}^{\mu \nu}=\frac{4 x^{2}}{Q^{2}} P^{\mu} P^{\nu}
$$

and defining the metric and longitudinal components of the total cross section

$$
\Delta \sigma \Delta=\Delta \sigma^{M} \Delta+\Delta \sigma^{L} \Delta,
$$

we obtain

$$
\begin{aligned}
& \frac{d \Delta \sigma^{M} \Delta}{d x d y d z}=Y^{M} \sum_{i=q, \bar{q}} 2 c_{i}\left\{\int \int _ { A } \frac { d u } { u } \frac { d \rho } { \rho } \left\{\Delta q_{i}\left(\frac{x}{u}\right) \Delta D_{q_{i}}\left(\frac{z}{\rho}\right) \delta(1-u) \delta(1-\rho)\right.\right. \\
& +\Delta q_{i}\left(\frac{x}{u}\right) \Delta D_{q_{i}}\left(\frac{z}{\rho}\right) \frac{\alpha_{s}}{2 \pi} \\
\times & {\left[-\frac{1+\epsilon}{\hat{\epsilon}}\left(\Delta P_{q \leftarrow q}(\rho) \delta(1-u)+\Delta P_{q \leftarrow q}(u) \delta(1-\rho)\right)+C_{f} \Delta \Phi^{M} \Delta_{q q}(u, \rho)\right] } \\
& +\Delta q_{i}\left(\frac{x}{u}\right) \Delta D_{g}\left(\frac{z}{\rho}\right) \frac{\alpha_{s}}{2 \pi} \\
\times & {\left[-\frac{1+\epsilon}{\hat{\epsilon}}\left(\Delta P_{g \leftarrow q}(\rho) \delta(1-u)+\Delta \hat{P} \Delta_{g q \leftarrow q}(u) \delta(\rho-a)\right)+C_{f} \Delta \Phi^{M} \Delta_{q g}(u, \rho)\right] } \\
& +\Delta g\left(\frac{x}{u}\right) \Delta D_{q_{i}}\left(\frac{z}{\rho}\right) \frac{\alpha_{s}}{2 \pi} \\
\times & {\left.\left[-\frac{1+\epsilon}{\hat{\epsilon}}\left(\Delta P_{q \leftarrow g}(u) \delta(1-\rho)+\Delta \hat{P} \Delta_{q \bar{q} \leftarrow g}(u) \delta(\rho-a)\right)+T_{f} \Delta \Phi^{M} \Delta_{g q}(u, \rho)\right]\right\} } \\
& +\int_{B} \frac{d u}{u}(1-x)\left\{\Delta M \Delta_{q_{i}}\left(\frac{x}{u},(1-x) z\right) \delta(1-u)\right.
\end{aligned}
$$




$$
\begin{aligned}
& +\Delta M \Delta_{q_{i}}\left(\frac{x}{u},(1-x) z\right) \frac{\alpha_{s}}{2 \pi}\left[-\frac{1-\epsilon}{\hat{\epsilon}} P_{q \leftarrow q}(u)+C_{f}\right] \\
& \left.\left.+\Delta M \Delta_{g}\left(\frac{x}{u},(1-x) z\right) \frac{\alpha_{s}}{2 \pi}\left[-\frac{1-\epsilon}{\hat{\epsilon}} P_{q \leftarrow g}(u)+T_{f} \Delta \Phi^{M} \Delta_{g}(u)\right]\right\}\right\}
\end{aligned}
$$

and

$$
\begin{aligned}
\frac{d \Delta \sigma^{L} \Delta}{d x d y d z} & =Y^{L} \sum_{i=q, \bar{q}} 2 c_{i}\left\{\int \int _ { A } \frac { d u } { u } \frac { d \rho } { \rho } \left\{\Delta q_{i}\left(\frac{x}{u}\right) \Delta D_{q_{i}}\left(\frac{z}{\rho}\right) \frac{\alpha_{s}}{2 \pi} C_{f} \Delta \Phi^{L} \Delta_{q q}(u, \rho)\right.\right. \\
& +\Delta q_{i}\left(\frac{x}{u}\right) \Delta D_{g}\left(\frac{z}{\rho}\right) \frac{\alpha_{s}}{2 \pi} C_{f} \Delta \Phi^{L} \Delta_{q g}^{A}(u, \rho) \\
& \left.+\Delta g\left(\frac{x}{u}\right) \Delta D_{q_{i}}\left(\frac{z}{\rho}\right) \frac{\alpha_{s}}{2 \pi} T_{f} \Delta \Phi^{L} \Delta_{g q}(u, \rho)\right\} \\
& +\int_{B} \frac{d u}{u}(1-x)\left\{\Delta M \Delta_{q_{i}}\left(\frac{x}{u},(1-x) z\right) \frac{\alpha_{s}}{2 \pi} C_{f} \Delta \Phi^{L} \Delta_{q}(u)\right. \\
& \left.\left.+\Delta M \Delta_{g}\left(\frac{x}{u},(1-x) z\right) \frac{\alpha_{s}}{2 \pi} T_{f} \Delta \Phi^{L} \Delta_{g}(u)\right\}\right\}
\end{aligned}
$$

While the longitudinal component, which is zero at leading order, is finite, the collinearly divergent metric component has yet to be subtracted, using the method indicated in the last subsection. We then use the NLO definition of polarized parton distributions [15, 14] and polarized fragmentation functions in the $\overline{M S_{p}}$ scheme, and introduce the NLO doubly polarized fracture functions by means of

$$
\begin{gathered}
\Delta M \Delta_{q_{i}}(\xi, \zeta)=\int_{\frac{\xi}{1-\zeta}}^{1} \frac{d u}{u}\left\{\left[\delta(1-u)+\frac{\alpha_{s}}{2 \pi} \frac{1+\epsilon}{\hat{\epsilon}} P_{q \leftarrow q}(u)\right] \Delta M \Delta_{q_{i}}\left(\frac{\xi}{u}, \zeta, Q^{2}\right)\right. \\
\left.+\frac{\alpha_{s}}{2 \pi} \frac{1+\epsilon}{\hat{\epsilon}} P_{q \leftarrow g}(u) \Delta M \Delta_{g}\left(\frac{\xi}{u}, \zeta, Q^{2}\right)\right\} \\
+\int_{\xi}^{\frac{\xi}{\xi+\zeta}} \frac{d u}{u} \frac{u}{x(1-u)}\left\{\frac{\alpha_{s}}{2 \pi} \frac{1-\epsilon}{\hat{\epsilon}} \Delta \hat{P} \Delta_{g q \leftarrow q}(u) \Delta q_{i}\left(\frac{\xi}{u}, Q^{2}\right) \Delta D_{g}\left(\frac{\zeta u}{\xi(1-u)}, Q^{2}\right)\right. \\
\left.+\frac{\alpha_{s}}{2 \pi} \frac{1-\epsilon}{\hat{\epsilon}} \Delta \hat{P} \Delta_{q \bar{q} \leftarrow g}(u) \Delta g\left(\frac{\xi}{u}, Q^{2}\right) \Delta D_{q_{i}}\left(\frac{\zeta u}{\xi(1-u)}, Q^{2}\right)\right\} .
\end{gathered}
$$

In this case no, finite terms are subtracted since the corrections for the fracture functions have exactly the same structure as those in the unpolarized case. In fact, as can be seen in the contribution from the box diagram, finite 
soft terms (which for the box diagram are just the hat terms) coming from the effects of the initial- and final-state polarization in the HVBM prescription cancel leaving no soft contributions proportional to $\delta(\rho-a)$.

We then obtain the following finite metric component of the cross section

$$
\begin{aligned}
\frac{d \Delta \sigma^{M} \Delta}{d x d y d z}= & \\
Y^{M} & \sum_{i=q, \bar{q}} 2 c_{i}\left\{\int \int _ { A } \frac { d u } { u } \frac { d \rho } { \rho } \left\{\Delta q_{i}\left(\frac{x}{u}, Q^{2}\right) \Delta D_{q_{i}}\left(\frac{z}{\rho}, Q^{2}\right) \delta(1-u) \delta(1-\rho)\right.\right. \\
+ & \Delta q_{i}\left(\frac{x}{u}, Q^{2}\right) \Delta D_{q_{i}}\left(\frac{z}{\rho}, Q^{2}\right) \\
& \times \frac{\alpha_{s}}{2 \pi} C_{f}\left[\Delta \Phi^{M} \Delta_{q q}(u, \rho)-\Delta \tilde{f}_{q}^{D}(\rho) \delta(1-u)-\Delta \tilde{f}_{q}^{F}(u) \delta(1-\rho)\right] \\
+ & \Delta q_{i}\left(\frac{x}{u}, Q^{2}\right) \Delta D_{g}\left(\frac{z}{\rho}, Q^{2}\right) \\
& \times \frac{\alpha_{s}}{2 \pi} C_{f}\left[\Delta \Phi^{M} \Delta_{q g}(u, \rho)-\Delta \tilde{f}_{g}^{D}(\rho) \delta(1-u)\right] \\
+ & \Delta g\left(\frac{x}{u}, Q^{2}\right) \Delta D_{q_{i}}\left(\frac{z}{\rho}, Q^{2}\right) \\
& \left.\times \frac{\alpha_{s}}{2 \pi} T_{f}\left[\Delta \Phi^{M} \Delta_{g q}(u, \rho)-\Delta \tilde{f}_{g}^{F}(u) \delta(1-\rho)\right]\right\} \\
& +\int_{B} \frac{d u}{u}(1-x)\left\{\Delta M \Delta_{q_{i}}\left(\frac{x}{u},(1-x) z, Q^{2}\right) \delta(1-u)\right. \\
+ & \Delta M \Delta_{q_{i}}\left(\frac{x}{u},(1-x) z, Q^{2}\right) \frac{\alpha_{s}}{2 \pi} C_{f} \Delta \Phi^{M} \Delta_{q}(u) \\
+ & \left.\left.\Delta M \Delta_{g}\left(\frac{x}{u},(1-x) z, Q^{2}\right) \frac{\alpha_{s}}{2 \pi} T_{f} \Delta \Phi^{M} \Delta_{g}(u)\right\}\right\}
\end{aligned}
$$

where the finite terms subtracted from the polarized parton distributions are

$$
\Delta \tilde{f}_{q}^{F}(u)=4(u-1) \text { and } \Delta \tilde{f}_{g}^{F}(u)=2(1-u)
$$

and the coefficients can be found in the Appendix.

Notice that both the single polarized (final-state) fracture function $M \Delta$ and the doubly polarized one $\Delta M \Delta$ are not mere artefacts motivated by 
factorization, but they are physically motivated parton densities related to target fragmentation. In the latter case they parametrize unpolarized parton densities of a nucleon that fragments into a hadron with a definite polarization state. In the former case, they involve polarized parton distributions of fragmented nucleons?.

At this stage, it is worth noticing that any factorization scheme can be chosen as long as the distributions used had been obtained by means of a consistent NLO analysis (with the corresponding splitting functions and coefficients in the same scheme). For instance, in the case of polarized parton distributions, NLO parametrizations can be obtained from ref. 19 in the $\overline{M S_{p}}$ scheme, or from refs. [20, 21], in a mixed factorization scheme where only the $\Delta \tilde{f}_{q}^{F}(u)=4(u-1)$ has been substracted $\left(\Delta \tilde{f}_{g}^{F}(u)=0\right.$ in eq. (40)). In the case of polarized fragmentation functions, no parametrizations are available yet, but the same concepts apply to them, both in the case of coputations using dimensional regularization or off-shell gluons, as in ref. [13], to regularize the divergencies. The corresponding NLO polarized splitting functions have been obtained very recently [22] in the mixed factorization scheme $\left(\Delta \tilde{f}_{q}^{D}(y)=4(y-1)\right.$ and $\Delta \tilde{f}_{g}^{D}(u)=0$ in eq. (14)) and will allow for a complete NLO analysis of polarized fragmentation functions.

\section{Summary and Conclusions}

Summarizing our results, we have presented here the full $\mathcal{O}\left(\alpha_{s}\right)$ QCD corrections to the semi-inclusive production cross section of polarized hadrons in $e^{+} e^{-}$annihilation processes and polarized deep inelastic lepton-hadron scattering using dimensional regularization and the HVBM scheme.

Generalizing the factorization prescription that excludes soft contributions in polarized deep inelastic $\left(\overline{M S_{p}}\right)$ to polarized fragmentation functions and to two new types of fracture functions, we have shown that this prescription smoothly subtracts all the collinear divergences and soft contributions

\footnotetext{
${ }^{3}$ The notation used may be, up to a point, misleading: "double" and "single" refer to a given combination of cross sections that will be proportional, in the end, to one or the other fracture function. The partonic pictures for fracture functions are given by eqs. (25) and (33), also confirmed by the kernels that drive their evolution (polarized or unpolarized) in eqs. (27) and (36).
} 
present in the semi-inclusive production of polarized hadrons in polarized DIS without introducing kinematical cuts or other process-dependent procedures. We present for completeness also our results in the more familiar $\overline{M S}$ scheme, that offer the same advantages with respect to factorization, but with a less trivial interpretation for parton distributions.

In the case of polarized fragmentation functions, we find our results compatible with the ones presented in ref. [13], computed using a different regularization prescription. Of course, both results can be consistently used as long as the scheme dependence is cancelled by the use of the corresponding NLO splitting functions in the chosen scheme.

The evolution kernels obtained here for fracture functions also allow to study the pertubative component of the scale dependence, at least to leading order, of these densities, unveiling new aspects of target fragmentation processes induced by DIS. In this way we produce a fully consistent, physically motivated and complete set of QCD-corrected cross sections, of interest in future phenomenological approaches and forthcoming experiments. 


\section{Acknowledgements}

We gratefully acknowledge C. García Canal, G. Veneziano and D. Graudenz for enlightening comments and discussions.

\section{Appendix}

We append here the coefficients, some definitions and useful relations.

$$
\begin{aligned}
\text { a. Coefficients for single polarization } & \\
& \Phi \Delta_{q q}(u, \rho)= \\
- & 8 \delta(1-u) \delta(1-\rho)-\frac{1}{(1-u)_{+}}(1+\rho)-\frac{1}{(1-\rho)_{+}}(1+u) \\
+ & \delta(1-u)\left[\rho-1-(1+\rho) \ln (1-\rho)+\frac{1+\rho^{2}}{1-\rho} \ln \rho+2\left(\frac{\ln (1-\rho)}{1-\rho}\right)_{+}\right] \\
+ & \delta(1-\rho)\left[1-u-(1+u) \ln (1-u)+\frac{1+u^{2}}{1-u} \ln \frac{1-x}{u-x}+2\left(\frac{\ln (1-u)}{1-u}\right)_{+}\right] \\
+ & 2 \frac{1}{(1-\rho)_{+}} \frac{1}{(1-u)_{+}}+2(2+u)(1-x)-2(2-\rho)(1-x)^{2} \\
+ & \frac{x\left(6 x(1-\rho)(1-x)+x(1-\rho)-2+2 \rho\left(1-x^{2}\right)\right)}{u-x} \\
+ & \frac{(1-\rho)(1-x) x^{2}(2 x-1)}{(u-x)^{2}}
\end{aligned}
$$

$$
\begin{aligned}
\Phi \Delta_{q g}(u, \rho) & =\delta(1-u)(2-\rho) \ln [(1-\rho) \rho]+\left(\frac{1}{1-u}\right)_{+}(2-\rho) \\
& +\delta(\rho-a)(1+u) \ln \frac{1-u}{u}+\left(\frac{1}{\rho-a}\right)_{+}(1+u) \\
& -2(1-\rho)^{2}\left(1+\rho^{2}\right) \delta(\widehat{1-u})-1+\frac{2(1-\rho)(2 x-1)}{1-x} \\
& +\frac{u-2 u^{2}(1-x)}{u-x}-\frac{2(1-\rho)(1-u) x u(1-x)}{(u-x)^{2}}
\end{aligned}
$$




$$
+\frac{2(1-\rho)(1-u) x^{2}}{(1-x)(u-x)}+\frac{(1-\rho)(1-u) x^{2}}{(u-x)^{2}}-2 \delta(\widehat{\rho-} a)
$$

$$
\begin{aligned}
\Phi \Delta_{g q}(u, \rho) & =\delta(1-\rho)\left[1+\left(1-2 u+2 u^{2}\right)\left(-1+\ln \frac{(1-u)(1-x)}{u-x}\right)\right] \\
& +\left(\frac{1}{1-\rho}\right)_{+}\left(1-2 u+2 u^{2}\right)+2(1-u) \delta(\widehat{\rho-a}) \\
& +\delta(\rho-a)\left[1-\left(1-2 u+2 u^{2}\right)\left(-1+\ln \frac{1-u}{u}\right)\right] \\
& -\left(\frac{1}{\rho-a}\right)_{+}\left(1-2 u+2 u^{2}\right)
\end{aligned}
$$

$$
\begin{gathered}
\Phi \Delta_{q}(u)=-\frac{1+u^{2}}{1-u} \ln (u)-(1+u) \ln (1-u)+2\left(\frac{\ln (1-u)}{(1-u)}\right)_{+} \\
-\frac{3}{2}\left(\frac{1}{1-u}\right)_{+}+3 u+\left(-\frac{9}{2}-\frac{\pi^{2}}{3}\right) \delta(1-u)-2(\widehat{1-u}) \\
\Phi \Delta_{g}(u)=2(\widehat{1-u})+(2 u-1)\left[\ln \left(\frac{1-u}{u}\right)-1\right]
\end{gathered}
$$

b. Coefficients for double polarization

i. Metric

$$
\begin{aligned}
& \Delta \Phi^{M} \Delta_{q q}(u, \rho)= \\
- & 8 \delta(1-u) \delta(1-\rho)-\frac{1}{(1-u)_{+}}(1+\rho)-\frac{1}{(1-\rho)_{+}}(1+u) \\
+ & \delta(1-u)\left[\rho-1-(1+\rho) \ln (1-\rho)+\frac{1+\rho^{2}}{1-\rho} \ln \rho+2\left(\frac{\ln (1-\rho)}{1-\rho}\right)_{+}\right] \\
+ & \delta(1-\rho)\left[u-1-(1+u) \ln (1-u)+\frac{1+u^{2}}{1-u} \ln \frac{1-x}{u-x}+2\left(\frac{\ln (1-u)}{1-u}\right)_{+}\right]
\end{aligned}
$$




$$
\begin{aligned}
& +2 \frac{1}{(1-\rho)_{+}} \frac{1}{(1-u)_{+}}+\frac{(1-\rho) x(2 u-x-u x)}{(u-x)^{2}} \\
& +\frac{2(u-x-u x)}{u-x}
\end{aligned}
$$

$$
\begin{aligned}
\Delta \Phi^{M} \Delta_{q g}(u, \rho) & =\delta(1-u)(2-\rho) \ln [(1-\rho) \rho]+\left(\frac{1}{1-u}\right)_{+}(2-\rho) \\
& +\delta(\rho-a)(1+u)\left[2+\ln \frac{1-u}{u}\right]+\left(\frac{1}{\rho-a}\right)_{+}(1+u) \\
& -2\left(1-\rho^{2}\right) \delta(\widehat{-} u)-2(1-x)+\frac{(1-\rho) x}{u-x}-\frac{\rho(1-x) x}{u-x} \\
& +\frac{(1-\rho)(1-x) x^{2}}{(u-x)^{2}}+\frac{x^{2}}{u-x}-2 u \delta(\widehat{\rho-a})
\end{aligned}
$$

$$
\begin{aligned}
\Delta \Phi^{M} \Delta_{g q}(u, \rho) & =\delta(1-\rho)(2 u-1)\left(\ln \frac{(1-u)(1-x)}{u-x}\right) \\
& +\left(\frac{1}{1-\rho}\right)_{+}(2 u-1)-\left(\frac{1}{\rho-a}\right)_{+}(2 u-1) \\
& +\delta(\rho-a)(2 u-1)\left(-2-\ln \frac{1-u}{u}\right) \\
& +2(1-u) \delta(\widehat{1-\rho})
\end{aligned}
$$

$$
\begin{aligned}
& \Delta \Phi^{M} \Delta_{q}(u)=-\frac{1+u^{2}}{1-u} \ln (u)-(1+u) \ln (1-u)+2\left(\frac{\ln (1-u)}{1-u}\right)_{+} \\
&-\frac{3}{2}\left(\frac{1}{1-u}\right)_{+}+3-u+\left(-\frac{9}{2}-\frac{\pi^{2}}{3}\right) \delta(1-u) \\
& \Delta \Phi^{M} \Delta_{g}(u)=\left(1-2 u+2 u^{2}\right)\left(\ln \frac{1-u}{u}-1\right)
\end{aligned}
$$

ii. Longitudinal

$$
\Delta \Phi^{L} \Delta_{q q}(u, \rho)=\frac{2(\rho-a) u^{3}(1-x)^{3}}{(u-x)^{2}}
$$




$$
\begin{gathered}
\Delta \Phi^{L} \Delta_{q g}(u, \rho)=-2(1-\rho) u^{3} \frac{(1-x)^{2}}{(u-x)^{2}} \\
\Delta \Phi^{L} \Delta_{g q}(u, \rho)=0 \\
\Delta \Phi^{L} \Delta_{q}(u)=u \\
\Delta \Phi^{L} \Delta_{g}(u)=2 u(1-u)
\end{gathered}
$$

\section{c. Splitting functions}

We include here the expressions for the virtual [17] and real [18] splitting functions:

$$
\begin{aligned}
\Delta P_{q \leftarrow q}(u) & =P_{q \leftarrow q}(u)=C_{f}\left[2\left(\frac{1}{1-u}\right)_{+}+\frac{3}{2} \delta(1-u)-1-u\right] \\
\Delta P_{q \leftarrow g}(u) & =\Delta \hat{P}_{q \bar{q} \leftarrow g}(u)=-\Delta \hat{P} \Delta_{q \bar{q} \leftarrow g}(u)=T_{f}[2 u-1] \\
P_{q \leftarrow g}(u) & =-\hat{P} \Delta_{q \bar{q} \leftarrow g}(u)=T_{f}\left[2 u^{2}-2 u+1\right] \\
\Delta P_{g \leftarrow q} & =\hat{P} \Delta_{g q \leftarrow q}(1-u)=\Delta \hat{P} \Delta_{g q \leftarrow q}(1-u)=C_{f}[2-u] \\
P_{g \leftarrow q}(u) & =\Delta \hat{P}_{g q \leftarrow q}(1-u)=C_{f}\left[2 \frac{1}{u}-2+u\right]
\end{aligned}
$$

\section{d. Coefficients for single initial-state polarization}

We include here the coefficients from ref. 10 adapted to the definition of the ()$_{+}$prescriptions used in ref. [8]:

$$
\begin{aligned}
& \Delta \Phi_{q q}(u, \rho)= \\
- & 8 \delta(1-\rho) \delta(1-u)-\left(\frac{1}{1-\rho}\right)_{+}(1+u)-\left(\frac{1}{1-u}\right)_{+}(1+\rho) \\
+ & {\left[(1-\rho)+\frac{1+\rho^{2}}{1-\rho} \ln \rho-(1+\rho) \ln (1-\rho)+2\left(\frac{\ln (1-\rho)}{1-\rho}\right)_{+}\right] \delta(1-u) } \\
+ & {\left.\left[-(1-u)+\frac{1+u^{2}}{1-u} \ln \left(\frac{1-x}{u-x}\right)-(1+u) \ln (1-u)+2\left(\frac{\ln (1-u)}{1-u}\right)\right]_{+}\right] \delta(1-\rho) }
\end{aligned}
$$




$$
\begin{aligned}
& -\frac{(1-\rho)(1-u+(1-x)(-1+u(1+2 u)(1-x)-x))}{(u-x)^{2}}+\frac{4 u(1-x)}{u-x} \\
& -\frac{2(1-u) u(1-x)}{u-x}-\frac{2 x}{u-x}+2\left(\frac{1}{1-\rho}\right)_{+}\left(\frac{1}{1-u}\right)_{+}-2(1-u) \delta(\widehat{1-\rho})
\end{aligned}
$$

$$
\begin{aligned}
\Delta \Phi_{q g}(u, \rho) & =\delta(1-u)\left[\rho+\left(\rho+\frac{2}{\rho}-2\right) \ln (\rho(1-\rho))\right] \\
& +\delta(\rho-a)\left[-2(1-u)-(1-u)+\frac{1+u^{2}}{1-u} \ln \frac{1-u}{u}\right]-\frac{2(1-\rho)^{2}}{\rho(1-u)} \\
& +\frac{-2 u}{(\rho-a)(1-u)}-\frac{2(1-u) u(1-x)}{u-x}+\frac{2(1-\rho)^{2} u^{3}(1-x)^{2}}{(\rho-a)(1-u)(u-x)^{2}} \\
& +\frac{(2-\rho) x}{u-x}+\left(\frac{1}{\rho-a}\right)+\frac{1+u^{2}}{1-u}+\left(\frac{1}{1-u}\right)_{+}\left(\rho+\frac{2}{\rho}-2\right) \\
& +\frac{(1-\rho) u(1-x) x}{(u-x)^{2}}
\end{aligned}
$$

$$
\begin{aligned}
\Delta \Phi_{g q}(u) & =\delta(1-\rho)\left[2(\widehat{1-u})+(2 u-1) \ln \left(\frac{(1-x)(1-u)}{u-x}\right)\right] \\
& +\delta(\rho-a)\left[2(\widehat{-u})+(2 u-1) \ln \frac{1-u}{u}\right] \\
& +(2 u-1)\left[\left(\frac{1}{1-\rho}\right)_{+}+\left(\frac{1}{\rho-a}\right)_{+}\right]-2 u \frac{1-x}{u-x}
\end{aligned}
$$$$
\begin{aligned}
\Delta \Phi_{q}(u) & =-\frac{1+u^{2}}{1-u} \ln (u)-(1+u) \ln (1-u)+2\left(\frac{\ln (1-u)}{1-u}\right)_{+} \\
& -\frac{3}{2}\left(\frac{1}{1-u}\right)_{+}+3 u+\left(-\frac{9}{2}-\frac{\pi^{2}}{3}\right) \delta(1-u)-2(\widehat{1-u})
\end{aligned}
$$

$$
\Delta \Phi_{g}(u, \rho)=2(\widehat{-u})+(2 u-1)\left[\ln \left(\frac{1-u}{u}\right)-1\right]
$$




\section{References}

[1] M. Burkardt and R.L. Jaffe, Phys. Rev. Lett. 70, 2537 (1993).

[2] Compass Proposal, CERN/SPSLC 96-14, SPSC/P 297 (1996).

[3] R.L. Jaffe, MIT-CTP-2534 (1996) hep-ph/9605456.

[4] J. Ellis, D. Kharzeev and A. Kotzinian, Z. Phys. C69, 467 (1996).

[5] M. Anselmino, A. Efremov and E. Leader, Phys. Rep. 261 (1995).

[6] J. Ellis and M. Karliner, CERN-TH/95-334 (1995) hep-ph/9601280.

[7] L. Trentadue and G. Veneziano, Phys. Lett. B323, 201 (1994).

[8] D. Graudenz, Nucl. Phys. B432, 351 (1994).

[9] W. Vogelsang, Z. Phys. C50, 275 (1991).

[10] D. de Florian, C.A. García Canal and R. Sassot, Nucl. Phys. B470, 195 (1996).

[11] C.G. Bollini and J.J. Giambiaggi, Nuovo Cimento 12B, 20 (1972).

[12] G. 't Hooft and M. Veltman, Nucl. Phys. B44, 189 (1972);

P. Breitenlohner and D. Maison, Commun. Math. Phys. 52, 11 (1977).

[13] V. Ravindran, hep-ph/9607384, hep-ph/9606273, hep-ph/9606272.

[14] D. de Florian and R. Sassot, Phys. Rev. D51, 6052 (1995).

[15] L.E. Gordon and W. Vogelsang, Phys. Rev D48, 3136 (1993).

[16] A. Weber, Nucl. Phys. B382, 63 (1992).

[17] G. Altarelli and G. Parisi, Nucl. Phys. B126, 298 (1977).

[18] E. Konishi, A. Ukawa and G. Veneziano, Nucl. Phys. B157, 45 (1979).

[19] R.D. Ball, S. Forte and G. Ridolfi, Phys. Lett. B378, 255 (1996).

[20] T. Gehrmann and W.J. Stirling, Phys. Rev. D53, 6100 (1996). 
[21] M. Glück, E. Reya, M. Stratmann and W. Vogelsang, Phys. Rev. D53, 4775 (1996).

[22] M. Stratmann and W. Vogelsang, hep-ph/9612250 (1996). 

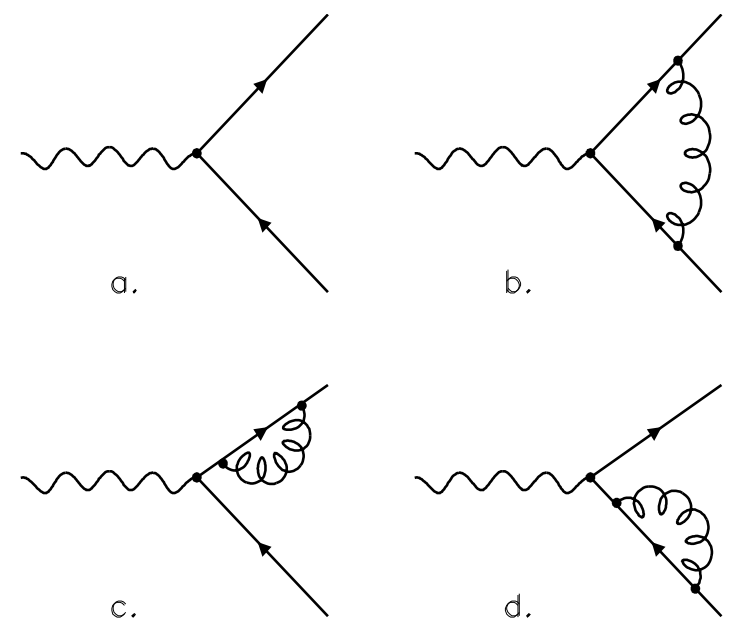

Figure 1: a: Lowest order diagram for $e^{+} e^{-}$anihilation; b, c, d: Virtual gluon corrections to $\mathbf{1 a}$
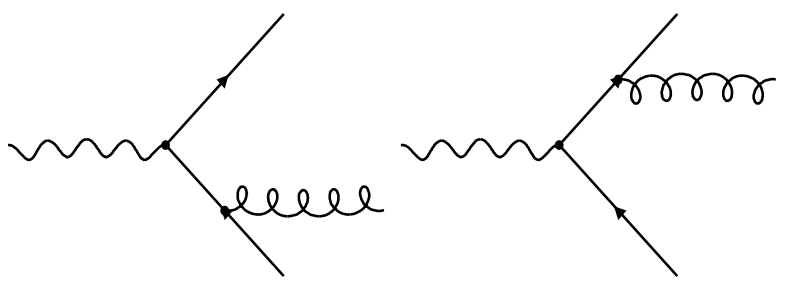

Figure 2: Real gluon emission corrections to $e^{+} e^{-}$anihilation 

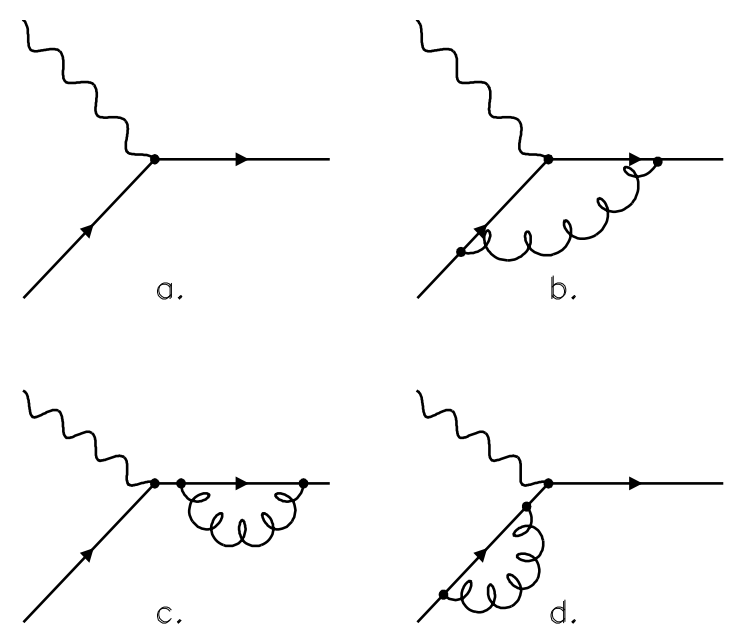

Figure 3: a: Lowest order parton-photon graph; b, c, d: Virtual gluon corrections graphs to $\mathbf{3 a}$
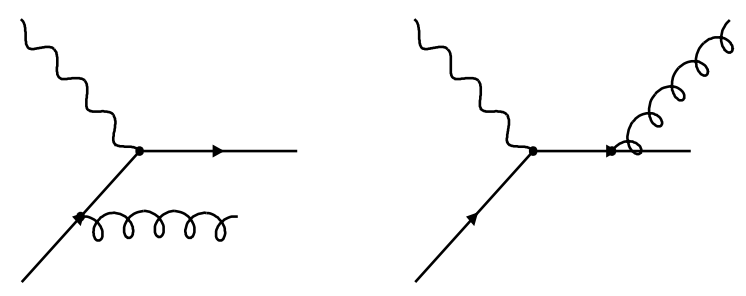

Figure 4: Real gluon emission corrections to DIS 

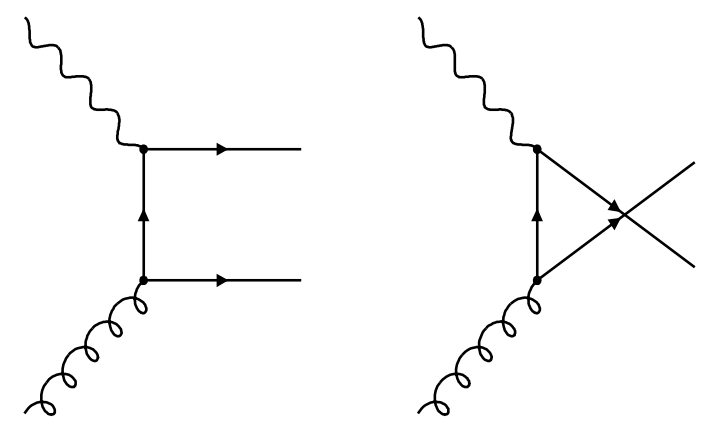

Figure 5: Gluon contribution (box diagram) to DIS 